\title{
Estructura de la comunidad de copépodos en Bahía Magdalena, México, durante El Niño 1997-1998
}

\author{
Copepod community structure in Magdalena Bay, Mexico, during El Niño 1997-1998 \\ Gladis A. López-Ibarra ${ }^{1}$ y Ricardo Palomares-García ${ }^{1}$
}

\author{
${ }^{1}$ Centro Interdisciplinario de Ciencias Marinas - Instituto Politécnico Nacional. Departamento de Plancton y Ecología Marina \\ A.P. 592, C.P. 23096, La Paz, Baja California Sur, México \\ gibarra@ipn.mx
}

\begin{abstract}
Resumen.- Se evaluaron los cambios estacionales en la comunidad de copépodos, asociados a los cambios ambientales provocados por El Niño 1997/98, durante cuatro muestreos oceanográficos, en 14 estaciones, en Bahía Magdalena. Se identificaron 66 especies de copépodos. En mayo de 1997, antes de la llegada de aguas cálidas al área, la comunidad estuvo constituida por una alta dominancia, baja diversidad y el predominio de dos especies de afinidad templada: Calanus pacificus y Labidocera trispinosa. Durante el período cálido (agosto y noviembre), las especies dominantes fueron de afinidad tropical, como Acartia clausi y A. lilljeborgii. En agosto, la diversidad presentó un incremento en las estaciones influenciadas por agua del exterior mientras que en noviembre, la diversidad aumentó en toda la bahía; en enero, la dominancia fue baja y la especie más abundante fue Paracalanus aculeatus, observándose un incremento generalizado de la diversidad en toda la bahía. Se identificaron dos ambientes: uno ligado a las aguas internas de la bahía, con predominio de especies residentes $y$ de aquellas que frecuentemente entran a la bahía por intercambio de marea y otro asociado con las estaciones influenciadas por aguas exteriores, con predominio de especies de ambientes oceánicos o neríticos. En esta última área se refleja, con mayor claridad, la llegada de aguas cálidas y su fauna asociada. Los cambios más relevantes en la comunidad de copépodos coincidieron con la presencia de anomalías positivas de temperatura. Los cambios más notables fueron: el incremento en un $75 \%$ de las especies de afinidad tropical, la presencia de Pontella fera, la cual no había sido reportada en la bahía y la sucesión de la especie templada $P$. parvus, por la especie tropical P. aculeatus.
\end{abstract}

Palabras clave: Afinidad biogeográfica, diversidad, evento de calentamiento

\begin{abstract}
Seasonal changes in copepods taxocoenosis associated to environmental changes caused by El Niño 1997/98 were evaluated during the four most intensive months of the event. Sixty six copepod species were identified. In May, before the arrival of warm waters to the area, the taxocoenosis structure was characterized by a high dominance, low diversity and the predominance of two species of temperated affinity: Calanus pacificus and Labidocera trispinosa. During the warm period (August and November), the dominant species were of tropical affinity, such as Acartia clausi and A. lilljeborgii. In August, diversity increased in the stations influenced by oceanic waters whereas in November, diversity increased in the entire bay; in January, the dominance was relative low and the most abundant tropical species was Paracalanus aculeatus, and a general increase of the diversity was observed in the entire bay. Two environmental zones in the bay were identified: one characterized by internal waters of the bay, with resident species and with those species frequently entering the bay by tidal currents, and another zone characterized by the influence of outside oceanic waters, with the dominance of oceanic or neritic species. In this last area, it is more clearly reflected the warm water arrival and its associated fauna. The most remarkable changes in the copepods community were coincident with the occurrence of positive temperature anomalies. Changes included a $75 \%$ increase of species of tropical affinity and the presence of Pontella fera, which had not been previously recorded in the area, and the replacement of the temperate species $P$. parvus, by $P$. aculeatus, a species of tropical affinity.
\end{abstract}

Key words: Biogeography affinity, diversity, warm event 


\section{Introducción}

Debido a que las comunidades planctónicas presentan una gran importancia en la transferencia de energía en los ambientes acuáticos, muchos estudios (Barber et al. 1985, Miller et al. 1985, Fiedler et al. 1986, Lavaniegos-Espejo et al. 2000) se han enfocado a la evaluación de los efectos que un evento El Niño puede provocar sobre estas comunidades, en particular, sobre las comunidades zooplanctónicas. Uno de los grupos más importantes por su diversidad y abundancia dentro del zooplancton es el de los copépodos, ya que pueden representar entre un $60 \%$ y un $80 \%$ de la biomasa zooplanctónica.

Los cambios en la estructura de la comunidad dependen, en buena medida, de la respuesta que tengan las poblaciones particulares ante los cambios ambientales, dependiendo de la magnitud del evento (Gómez-Gutiérrez et al. 1995, Lavaniegos-Espejo et al. 2002). En lo que respecta a la estructura de la comunidad de copépodos en particular, diversos estudios indican que ésta puede ser modificada por este tipo de fenómenos (Hernández-Trujillo 1999, Lavaniegos-Espejo et al. 2000, Lavaniegos-Espejo et al. 2002). Durante eventos El Niño, en aguas neríticas de la península de Baja California, se ha observado lo que algunos autores llaman una "tropicalización" en la comunidad de copépodos asociada a la llegada de aguas tropicales y su fauna característica (Hernández-Trujillo 1999, Lavaniegos-Espejo et al. 1998, LavaniegosEspejo et al. 2002). Esta condición va acompañada de una reducción en la abundancia de especies templadas, típicas de la Corriente de California.

A pesar que los efectos más evidentes de un evento El Niño son la disminución en la biomasa del fitoplancton y zooplancton, diversos estudios encuentran que la sucesión estacional de las especies dominantes de la comunidad de copépodos no se ha visto fuertemente afectada (Palomares-García \& Gómez-Gutiérrez, 1996), lo que sugiere una elevada capacidad de estas especies para adaptarse a los cambios en las condiciones ambientales. El objetivo de este trabajo fue determinar la variación en la estructura de la comunidad de copépodos en función de los cambios ambientales asociados con el evento El Niño 1997/98 en Bahía Magdalena. Esta idea se fundamenta en el hecho de que el evento El Niño 1997/98 se comportó de manera diferente a eventos previos en Bahía Magdalena. Las primeras anomalías positivas de temperatura se registraron en junio de 1997, a diferencia de El Niño 1982/83 que se inició en noviembre de 1982. Además, fue más intenso que el de 1982/83, al menos en su etapa inicial, aunque con una duración menor (aproximadamente 13 meses el de 1997/98 y 22 meses el de 1982/83). Considerando lo anterior, se planteó la hipótesis de que la aparición temprana y la intensidad del evento del Niño 1997/98, podría traducirse en cambios apreciables en la estructura de la comunidad de copépodos. Dichos cambios serían característicos de El Niño 1997/98 y diferentes a los reportados para eventos anteriores en Bahía Magdalena (Palomares-García 1992, Palomares-García \& Gómez-Gutiérrez 1996).

\section{Materiales y métodos}

\section{Muestreo de campo}

El material biológico se obtuvo en mayo, agosto y noviembre de 1997 y enero de 1998. Las muestras de zooplancton fueron recolectadas en 14 estaciones localizadas en Bahía Magdalena, ubicada en la costa occidental de la península de Baja California, entre los $24^{\circ} 15^{\prime}$ y los $25^{\circ} 20^{\prime} \mathrm{N}$ y entre los $111^{\circ} 30^{\prime}$ y los $112^{\circ}$ $15^{\prime} \mathrm{O}$, en el límite sur del sistema de la Corriente de California, en donde confluyen las masas de agua del Pacífico norte, Pacífico central y Pacífico oriental tropical (Roden 1971, Brinton \& Reid 1986) (Fig. 1); este sistema lagunar se divide en tres zonas hidrológicas: La zona norte o zona de canales, formada principalmente por esteros y canales con profundidad promedio de 3,5 m, la cual está rodeada por mangle (Avicennia geminans y Rhizophora mangle); la zona central, denominada bahía Magdalena, comunicada con el Océano Pacífico por una boca de $38 \mathrm{~m}$ de profundidad y $4 \mathrm{~km}$ de ancho y por último, la zona sur, denominada bahía Almejas, conectada al océano por un canal estrecho $(0,2 \mathrm{~km})$ y somero $(5-7 \mathrm{~m})$. El área de estudio fue solo Bahía Magdalena. Este sistema lagunar generalmente presenta condiciones anti-estuarinas, por la baja precipitación (70 $\mathrm{mm}$ anuales) y la escasa afluencia de agua dulce, así como una elevada tasa de evaporación (Álvarez-Borrego et al. 1975). Para recolectar las muestras se utilizó una red cónica de 60 cm de diámetro de boca y una abertura de malla de 333 $\mathrm{mm}$; los arrastres fueron superficiales, en círculo, con una duración de 5 minutos; todos los muestreos fueron diurnos. Los organismos recolectados se preservaron con formalina al $4 \%$ neutralizada con borato de sodio. Simultáneamente se tomaron registros de temperatura superficial del mar con un termómetro de cubeta. 


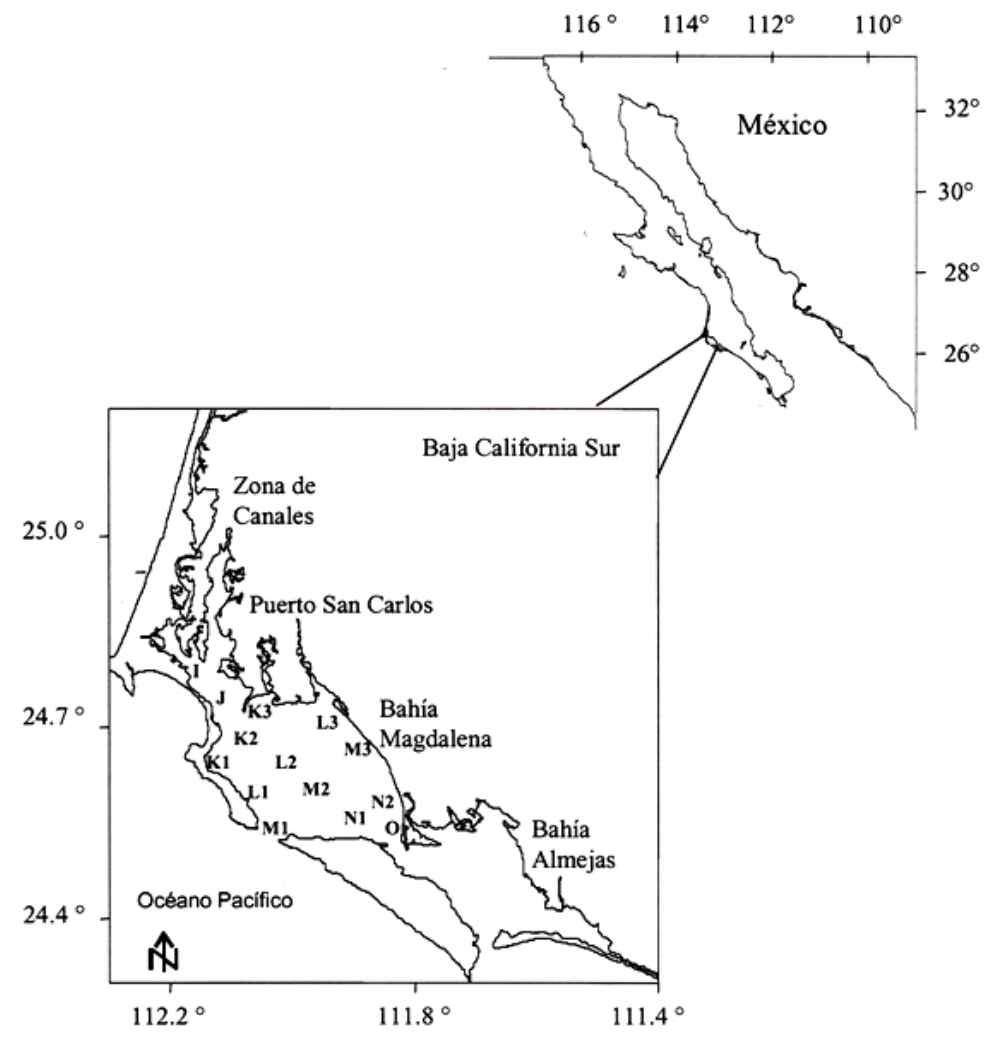

Figura 1

Área de estudio y estaciones de muestreo

Study area and sampling stations

\section{Análisis de las muestras}

La biomasa zooplanctónica se estimó mediante el método de volumen desplazado (Stedman 1976) y se obtuvieron submuestras de $1 / 2$ a $1 / 16$ con un fraccionador Folsom dependiendo del volumen original de la muestra original. Todas las muestras con un volumen menor a $10 \mathrm{~mL}$ se analizaron en su totalidad. La cuantificación de los copépodos adultos se realizó a partir de las submuestras; el número de organismos se estandarizó a $100 \mathrm{~m}^{3}$ de agua filtrada.

\section{Análisis de los datos}

Se determinó la composición y la abundancia de la comunidad de copépodos. Las especies dominantes se detectaron mediante curvas de K-dominancia, y con el propósito de conocer los rasgos estructurales, se estimó el índice de diversidad y equidad de Shannon (Pielou 1975); para obtener la afinidad biogeográfica de las especies de copépodos se realizó una búsqueda bibliográfica (Raymont 1983, Palomares-García et al. 1998). La serie de anomalías de temperatura para Bahía Magdalena (AT) desde enero de 1982 a septiembre de 1998, fueron tomadas y modificadas de PalomaresGarcía et al. (2003). Se realizaron análisis de ordenación mediante el análisis de componentes principales (ACP). Para este análisis se utilizaron únicamente aquellas especies que aparecieron en más de una estación estandarizando la matriz mediante una transformación logarítmica [log. $(\mathrm{X}+1)]$. Dicha transformación se aplicó para disminuir la magnitud de las diferencias de abundancia entre especies.

\section{Resultados}

\section{Temperatura superficial del mar y biomasa zooplanctónica}

La TSM para Bahía Magdalena (Fig. 2) mostró una tendencia estacional, con valores de $17-23^{\circ} \mathrm{C}$ en mayo 
de 1997 , de $29-32^{\circ} \mathrm{C}$ en agosto de 1997 , de $24-26^{\circ} \mathrm{C}$ en noviembre de 1997 y de $21-23^{\circ} \mathrm{C}$ en enero de 1998. Aunque el intervalo de variación fue más amplio (19$31^{\circ} \mathrm{C}$ ), también se registró la temperatura mas elevada para la zona, superior en $3,2^{\circ} \mathrm{C}$ a la máxima registrada durante el evento de 1982/83. Por otro lado, las anomalías de temperatura (AT) para Bahía Magdalena, evidencian la presencia de tres periodos de calentamiento importantes (Fig. 3). El primero, registrado en el segundo semestre de 1982, presentando la AT más alta en julio de $1983\left(+2,6^{\circ} \mathrm{C}\right)$ (PalomaresGarcía \& Gómez-Gutiérrez 1996). Un segundo evento registrado en 1986/87, considerado como un El Niño débil, debido a que las mayores AT observadas fueron de $1{ }^{\circ} \mathrm{C}$ (Gómez- Gutiérrez et al. 1995). Durante el evento de 1997/98, la AT más alta fue $+4,4^{\circ} \mathrm{C}$, registrada en agosto de 1997 y tuvo una duración aproximada de 15 meses (Gómez-Gutiérrez et al. 1999, Lavaniegos-Espejo et al. 2000, Palomares-García et al. 2001, Palomares-García et al. 2003).
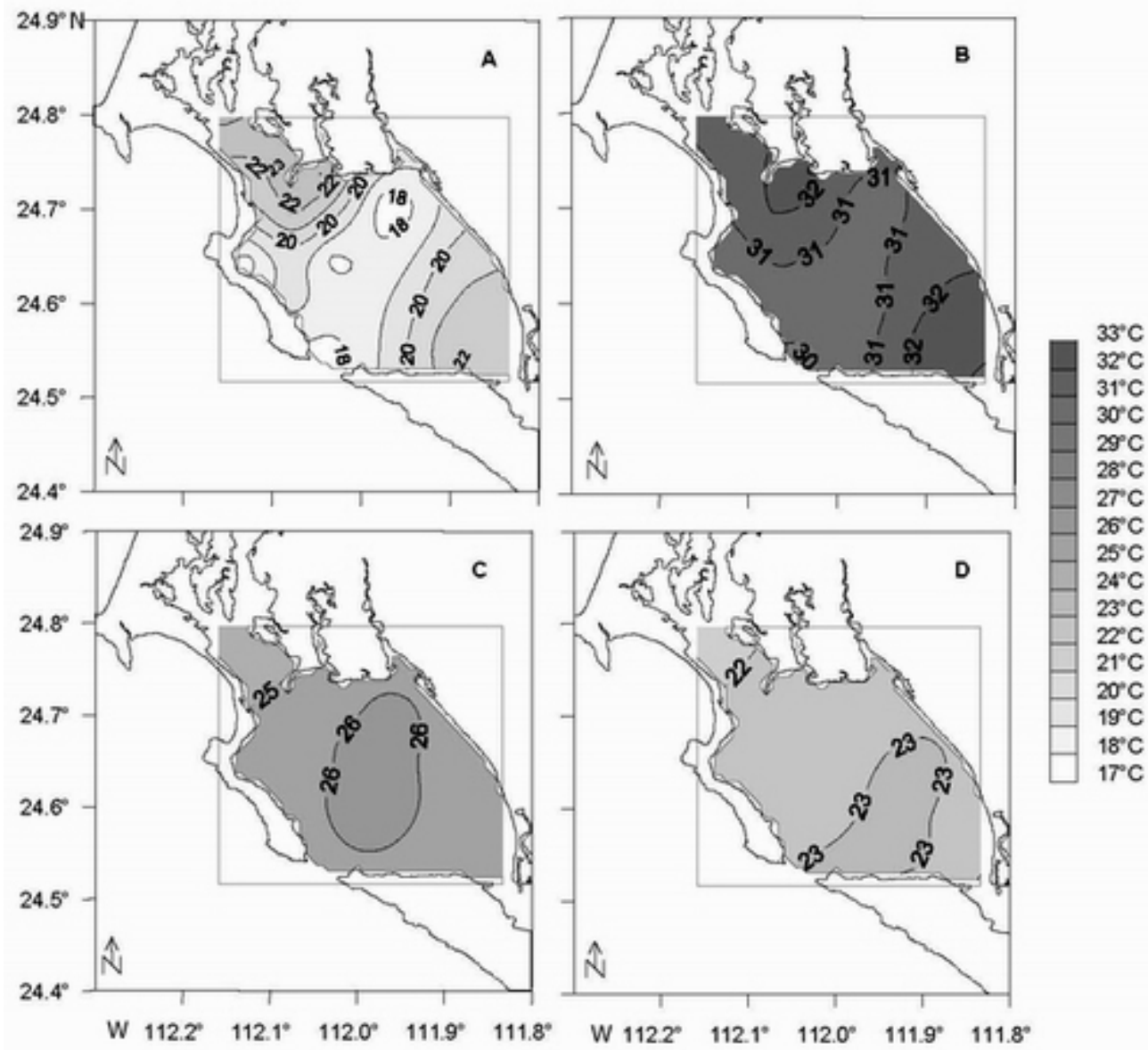

Figura 2

Temperatura superficial del mar en Bahía Magdalena durante El Niño 1997/98. A) mayo 1997, B) agosto 1997, C) noviembre 1997 y D) enero 1998

Sea surface temperature in Magdalena Bay during El Niño 1997/98. A) May 1997, B) August 1997, C) November 1997 and D) January 1998 


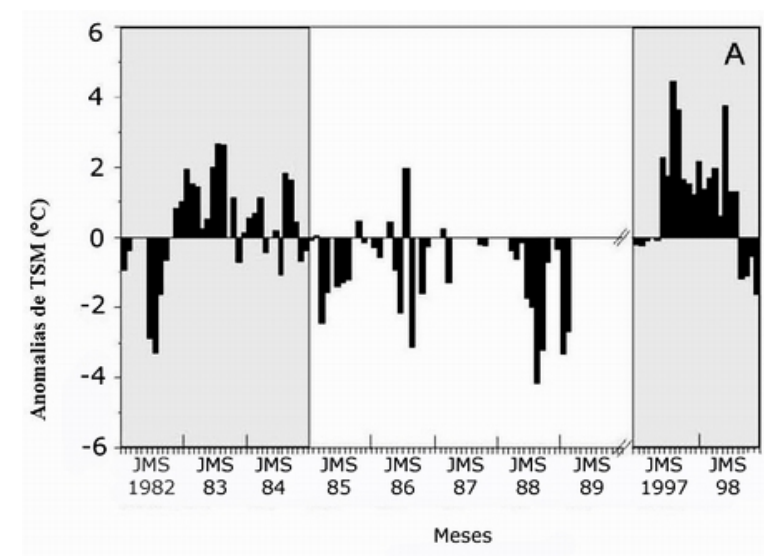

Figura 3

Anomalías de la temperatura superficial del mar desde enero de 1982 a septiembre de 1989 y desde mayo de 1997 a diciembre de 1998, en Bahía Magdalena, B.C.S., México (tomada y modificada de Palomares-García et al. 2003)

Sea surface temperature anomalies from January 1982 to

September 1989 and May 1997 to December 1998 in Magdalena Bay, B.C.S., Mexico (after Palomares-García et al. 2003)

La biomasa zooplanctónica también presentó un patrón estacional en la bahía, con un máximo de biomasa zooplanctónica en verano y un decremento hacia finales del invierno e inicios de la primavera. Este patrón se conserva tanto para años normales como en períodos con condiciones anómalas como El Niño o La Niña.

\section{Composición de especies y afinidad biogeográfica}

Durante el periodo de estudio se identificaron 30 géneros y 66 especies de copépodos pertenecientes a los órdenes Calanoida, Cyclopoida, Harpacticoida, Poecilostomatoida y Monstrilloida (Tabla 1). En mayo de 1997 se registró el número más bajo, con 22 especies pertenecientes a 16 géneros; en agosto de 1997, los números se incrementaron a 38 y 24 respectivamente; en noviembre de 1997 se encontraron 40 especies y 28 géneros y en enero de 1998 se registró el máximo número de especies con 44 y 24 géneros.

En cuanto a la afinidad biogeográfica de la comunidad de copépodos (Fig. 4), durante mayo de 1997 se observó un predominio de especies de afinidad templada cuando las temperaturas superficiales del mar fueron bajas, lo que coincidió con la mayor influencia de las aguas de la Corriente de California. En agosto y noviembre de 1997 y enero de 1998 se observó un

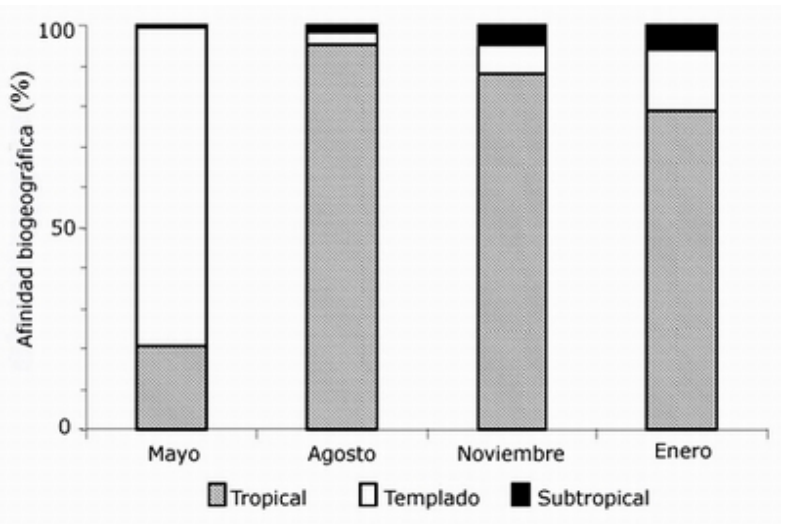

Figura 4

Afinidad biogeográfica de la comunidad de copépodos en Bahía Magdalena, B. C. S., México, en mayo 1997, agosto 1997, noviembre 1997 y enero 1998

Biogeographical affinity of the copepods community in Magdalena Bay, B.C.S., Mexico. in May 1997, August 1997, November 1997 and January 1998

marcado incremento de las especies de afinidad tropical, coincidiendo con el aumento de la temperatura superficial del mar, producto del avance de aguas cálidas durante el evento El Niño.

\section{Cambios en la abundancia, diversidad y dominancia de copépodos}

Los meses con mayor dominancia y baja diversidad fueron mayo y agosto de 1997, debido a que con solo tres y dos especies, respectivamente, acumularon el $90 \%$ de la abundancia. Las especies dominantes en ambos meses fueron diferentes, ya que en mayo, antes de la llegada de las aguas cálidas a la bahía, estuvieron presentes las especies de afinidad templada Calanus pacificus y Labidocera trispinosa, mientras que en agosto, las especies Acartia clausi y A. lilljeborgii, propias de ambientes lagunares, fueron las más abundantes. Además, se observó la presencia de un mayor número de especies de afinidad tropical y se registró la presencia de Pontella fera, que no había sido reportada anteriormente para esta zona. En noviembre y enero, la dominancia fue menor y la diversidad alta, ya que se necesitó de un mayor número de especies para sumar el $90 \%$ de la abundancia. Las especies más abundantes en noviembre pertenecieron al género Acartia, y en enero, al género Paracalanus (Fig. 5). 
Tabla 1

Abundancia promedio mensual de las especies registradas en Bahía Magdalena, B.C.S. México, durante El Niño 1997/98 (org. 100 m³) Monthly average abundance of recorded species in Magdalena Bay, B.C.S. Mexico, during El Niño 1997/98 (org. 100 m³)

\begin{tabular}{|c|c|c|c|c|c|c|}
\hline Especie & & Afinidad biogeográfica & Mayo & Agosto & Noviembre & Enero \\
\hline Acartia clausi & Giesbrecht, 1892 & Tem. - Cálida & 16424 & 1485838 & 92107 & 112151 \\
\hline Acartia danae & Giesbrecht, 1889 & Tropical & & 397644 & 3515 & 66133 \\
\hline Acartia lilljeborgii & Giesbrecht, 1889 & Ecuatorial & 7671 & 5129983 & 147820 & 133326 \\
\hline Acartia tonsa & Dana, 1849 & Tem. - Cálida & & & & 71 \\
\hline Acrocalanus gracilis & Giesbrecht, 1888 & Tropical & 9 & 6384 & 2411 & 5024 \\
\hline Calanus pacificus & Brodsky, 1948 & Templada & 40254 & 440 & 931 & 1100 \\
\hline Calocalanus pavo & Dana, 1849 & Tropical & & 129 & 155 & 84 \\
\hline Candacia aethiopica & Dana, 1849 & Tem. - Cálida & & 20 & & 17 \\
\hline Candacia catula & Giesbrecht, 1889 & Ecuatorial & & & & 16 \\
\hline Candacia curta & Dana, 1849 & Ecuatorial & 14 & & & \\
\hline Candacia longimana & Claus, 1863 & Trop. - Ecua. & & & 10 & \\
\hline Canthocalanus pauper & Giesbrecht, 1888 & Tem. - Cálida & & 1588 & 226 & \\
\hline Centropages abdominalis & Sato, 1913 & Tropical & & & & 7 \\
\hline Centropages calaninus & Dana, 1849 & Trop. - Ecua. & & 56 & & \\
\hline Centropages furcatus & Dana, 1849 & Tropical & & 26810 & 15640 & 11480 \\
\hline Centropages longicornis & Mori, 1932 & Tropical & & & & 17 \\
\hline Clausocalanus arcuicornis & Dana, 1849 & Trop. - Ecua. & 8 & 188 & 1061 & 6245 \\
\hline Clytemnestra rostrata & Brady, 1883 & Subtropical & & & 8 & \\
\hline Corycaeus catus & Dahl, 1894 & Tropical & & 592 & 141 & 284 \\
\hline Corycaeus latus & Dana, 1848 & Tropical & & & & 104 \\
\hline Corycaeus lautus & Dana, 1848 & Subtropical & & 264 & & 2671 \\
\hline Corycaeus robustus & Giesbrecht, 1891 & Tropical & 162 & 13576 & 2224 & 3286 \\
\hline Corycaeus speciosus & Dana, 1849 & Subtropical & 303 & 2441 & 1793 & 10815 \\
\hline Corycaeus andrewsi & Farran, 1911 & Tropical & 648 & 23378 & 4835 & 8966 \\
\hline Cymbasoma californiense & Suárez, 1999 & Tropical & & 56 & 34 & \\
\hline Euchaeta indica & Wolfenden, 1905 & Ecuatorial & & & & 52 \\
\hline Euchaeta longicornis & Giesbrecht, 1888 & Tropical & & & & 125 \\
\hline Euchaeta marina & Prestandrea, 1833 & Ecuatorial & & 142 & & 202 \\
\hline Euchaeta media & Giesbrecht, 1888 & Tropical & & & 37 & 18 \\
\hline Euterpina acutifrons & Dana, 1848 & Tropical & 23 & 1357 & 85 & 1028 \\
\hline Farranula gibbula & Giesbrecht, 1891 & Tropical & & 1106 & 343 & 5545 \\
\hline Labidocera acuta & Dana, 1849 & Ecuatorial & & 357 & 710 & 35 \\
\hline Labidocera acutifrons & Dana, 1849 & Tropical & & 80 & 275 & \\
\hline Labidocera jollae & Esterly, 1906 & Templada & 59 & 20 & & \\
\hline Labidocera trispinosa & Esterly, 1905 & Subtropical & 52914 & 188345 & 17716 & 41867 \\
\hline Lucicutia flavicornis & Claus, 1863 & Tropical & & & & 347 \\
\hline Nanocalanus minor & Claus, 1863 & Tropical & 3 & 2961 & 848 & 1727 \\
\hline Oithona plumifera & Baird, 1843 & Tropical & 11 & 1297 & 2690 & 10822 \\
\hline Oithona rigida & Giesbrecht, 1896 & Subtropical & 126 & 1554 & 5788 & 35056 \\
\hline Oithona similis & Claus, 1866 & Subtropical & & & 65 & 36 \\
\hline Oithona tenuis & Rosendorn, 1917 & Tropical & & & 233 & 86 \\
\hline Oncaea confiera & Giesbrecht, 1891 & Subtropical & & 693 & 383 & 1788 \\
\hline Oncaea venusta & Philippi, 1843 & Subtropical & & 453 & 1120 & 2074 \\
\hline Paracalanus aculeatus & Giesbrecht, 1888 & Subtropical & 39 & 6684 & 8232 & 174355 \\
\hline Paracalanus parvus & Claus, 1863 & Tem. - Cálida & & 3909 & 3242 & 64468 \\
\hline Pareucalanus sewelli & Fleminger, 1973 & Ecuatorial & & 1225 & 4047 & 4856 \\
\hline Pontella fera & Dana, 1849 & Tropical & & 20 & & \\
\hline Pontellina plumata & Dana, 1849 & Tropical & & & 13 & 17 \\
\hline Pontellopsis armata & Giesbrecht, 1889 & Tropical & & & 12 & \\
\hline Pseudodiaptomus wrighti & Johnson, 1964 & Tropical & 536 & 48863 & 8216 & 26628 \\
\hline Rhincalanus nasutus & Giesbrecht, 1888 & Templada & 69 & & & \\
\hline Saphirella tropica & Wolfenden, 1906 & Tropical & & & 10 & 7 \\
\hline Scolecithricella dentata & Giesbrecht, 1888 & Tem. - Cálida & 11 & & & \\
\hline Scolecithricella ctenopus & Giesbrecht, 1888 & Tropical & 28 & & & \\
\hline Scolecithrix bradyi & Giesbrecht, 1888 & Tropical & 9 & 165 & 10 & \\
\hline Subeucalanus subcrassus & Giesbrecht, 1888 & Ecuatorial & & 1642 & 1153 & 289 \\
\hline Temora discaudata & Giesbrecht, 1892 & Tropical & & 1168 & 1256 & 2573 \\
\hline Undinula vulgaris & Dana, 1852 & Tropical & 92 & 769 & 319 & 543 \\
\hline
\end{tabular}

Tem = templada; Trop $=$ tropical; Ecua $=$ ecuatorial 

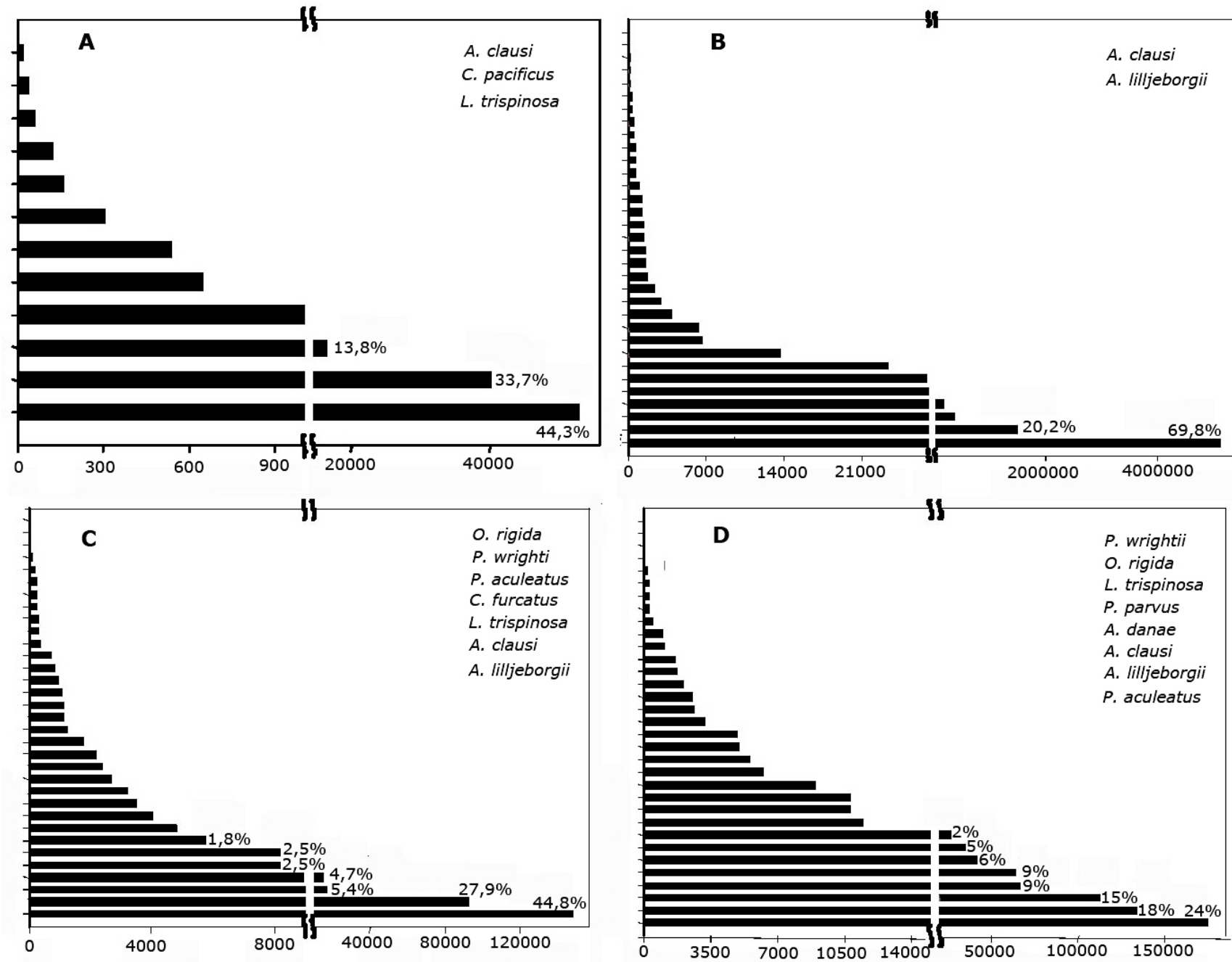

Abundancia específica (org. $100 \mathrm{~m}^{-3}$ )

Figura 5

Abundancia de especies dominantes de la comunidad de copépodos en relación con la temperatura superficial del mar, en Bahía Magdalena, B. C. S., México

Dominant species abundance of the copepod community in relation with the sea surface temperature in Magdalena Bay, B.C.S., Mexico

Durante mayo se presentó la menor diversidad (0,24 bits/ind.), mientras que los valores más elevados correspondieron a agosto (3,92 bits/ind.) coincidiendo con la llegada de aguas cálidas y su fauna asociada, por influencia del evento de El Niño.

Se observó una gran variación en la distribución espacial de la diversidad durante los cuatro meses de estudio (Fig. 6). Durante mayo se registró la menor diversidad para el área de la boca de la bahía; en agosto y noviembre, la diversidad fue menor en las estaciones del interior de la bahía y más alta en las estaciones influenciadas por las aguas cálidas exteriores. En enero de 1998, la diversidad fue más homogénea en toda la bahía, aunque también se observaron valores ligeramente más elevados en las estaciones cercanas a la boca. 


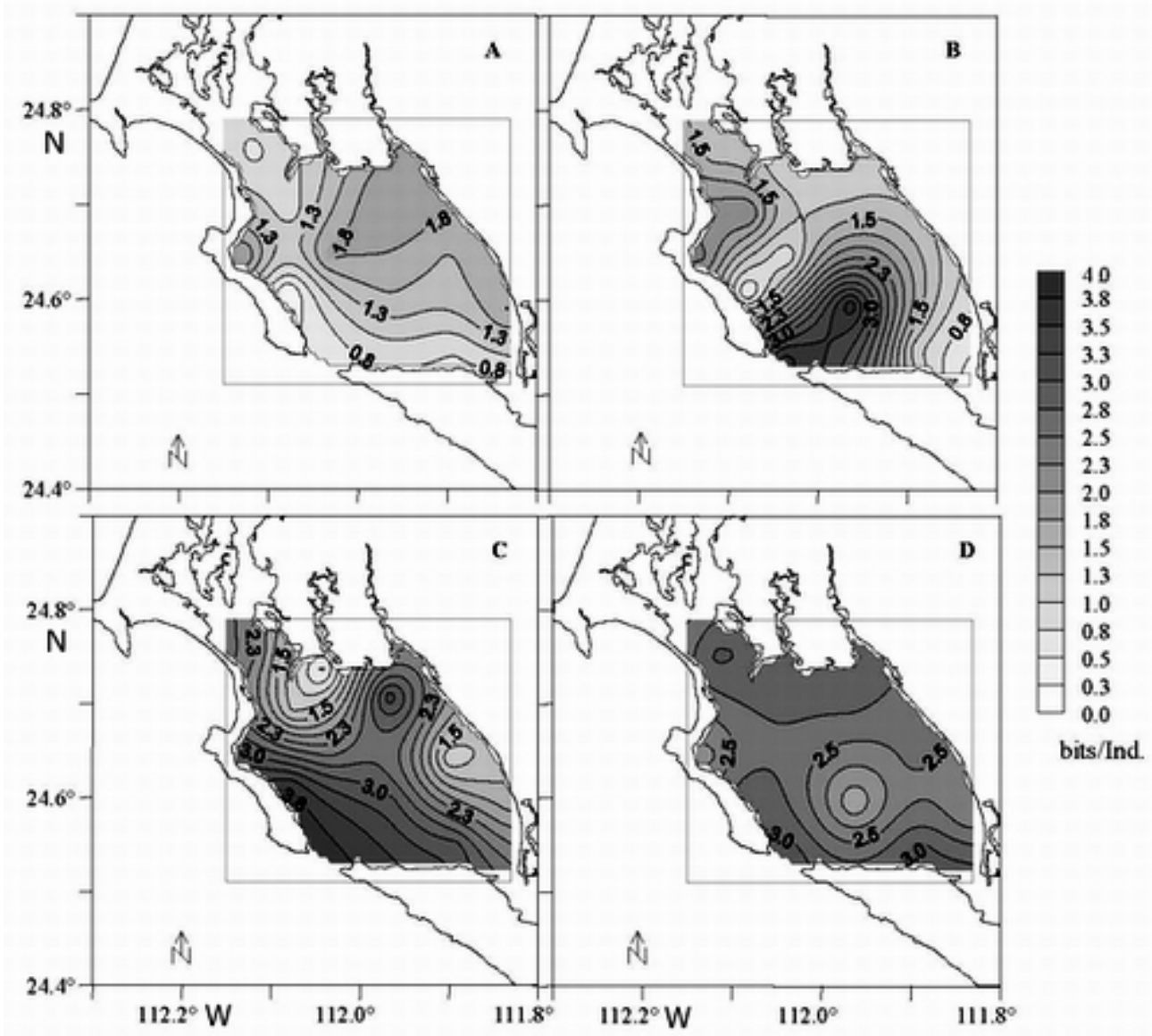

Figura 6

Distribución espacial de la diversidad de copépodos en Bahía Magdalena, B.C.S., México. A) mayo 1997, B) agosto 1997, C) noviembre 1997 y D) enero 1998

Spatial distribution of copepods diversity in Magdalena Bay, B. C. S., México.

A) May 1997, B) August 1997, C) November 1997 and D) January 1998

\section{Estructura de la comunidad y sucesión estacional de copépodos}

Para conocer las asociaciones de las especies de copépodos se aplicó un análisis de componentes principales (ACP). Los primeros dos componentes explicaron entre el 45 y el 55\% de la varianza, para los cuatro meses de estudio. El primer componente se asocia con el éxito poblacional de las especies que integran la comunidad en términos de su abundancia, en tanto que el segundo componente se relacionó con el origen de cada población y muestran la presencia de dos conjuntos de especies (Fig. 7) que son:

1. Especies residentes, que son frecuentes dentro de la bahía como Acartia clausi, A. lilljeborgii, Labidocera trispinosa, Centropages furcatus y Pseudodiaptomus wrighti. Estas especies pueden tener cambios estacionales en sus abundancias a lo largo del año, sin embargo, representan entre el $40-70 \%$ de la abundancia.

2. Especies neríticas y oceánicas que entran ocasionalmente a la bahía, como Calanus pacificus, Undinula vulgaris, Euchaeta marina, Calocalanus pavo, Oithona similis y O. tenuis. Estas especies presentan, por lo general, bajas abundancias en el interior de la bahía y se alternan dependiendo de los cambios estacionales de la Corriente de California y la Corriente Mexicana. Este último conjunto fue el más influenciado por el evento de El Niño 1997/98, observándose un incremento de su abundancia y frecuencia de aparición dentro de la bahía. 


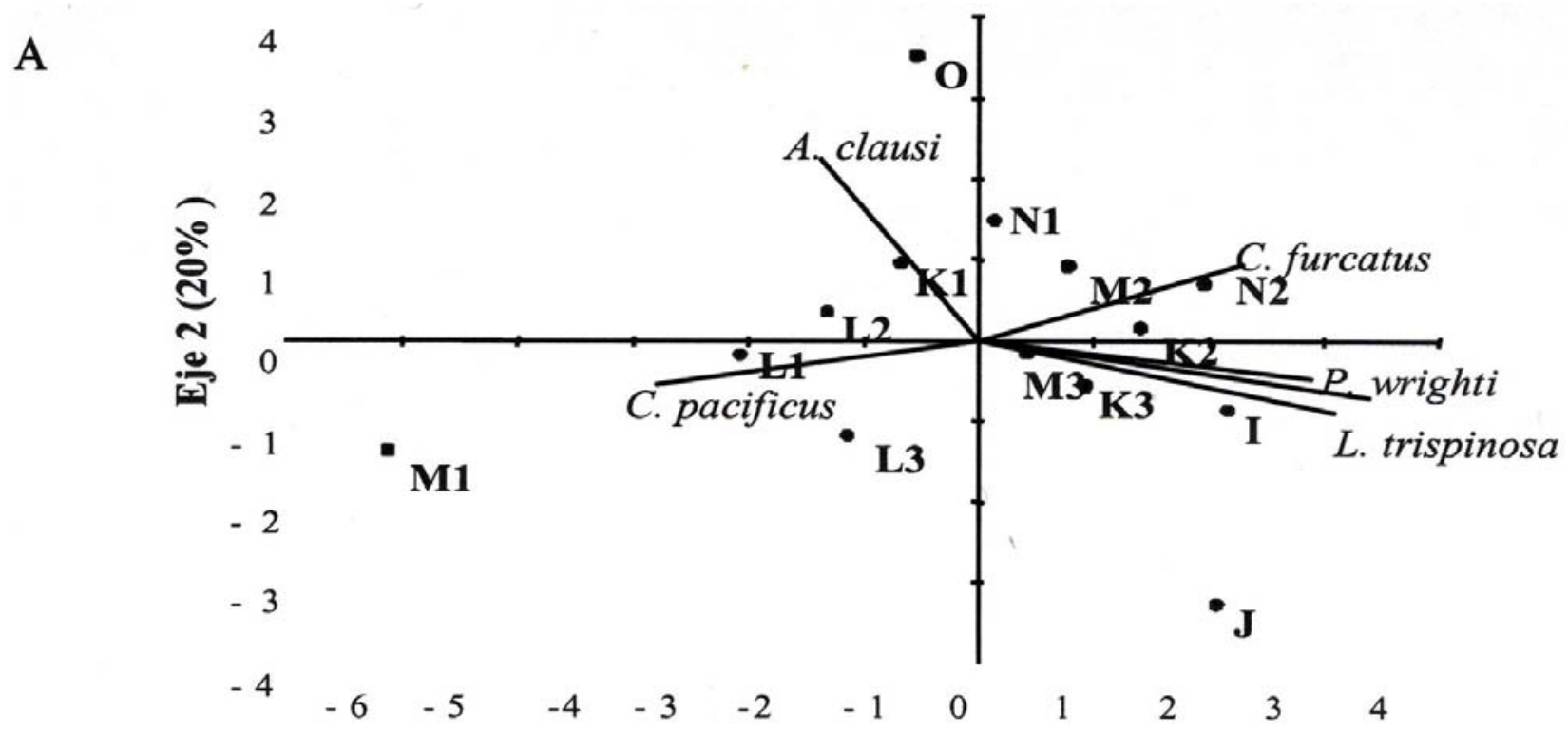

Eje $1(29 \%)$

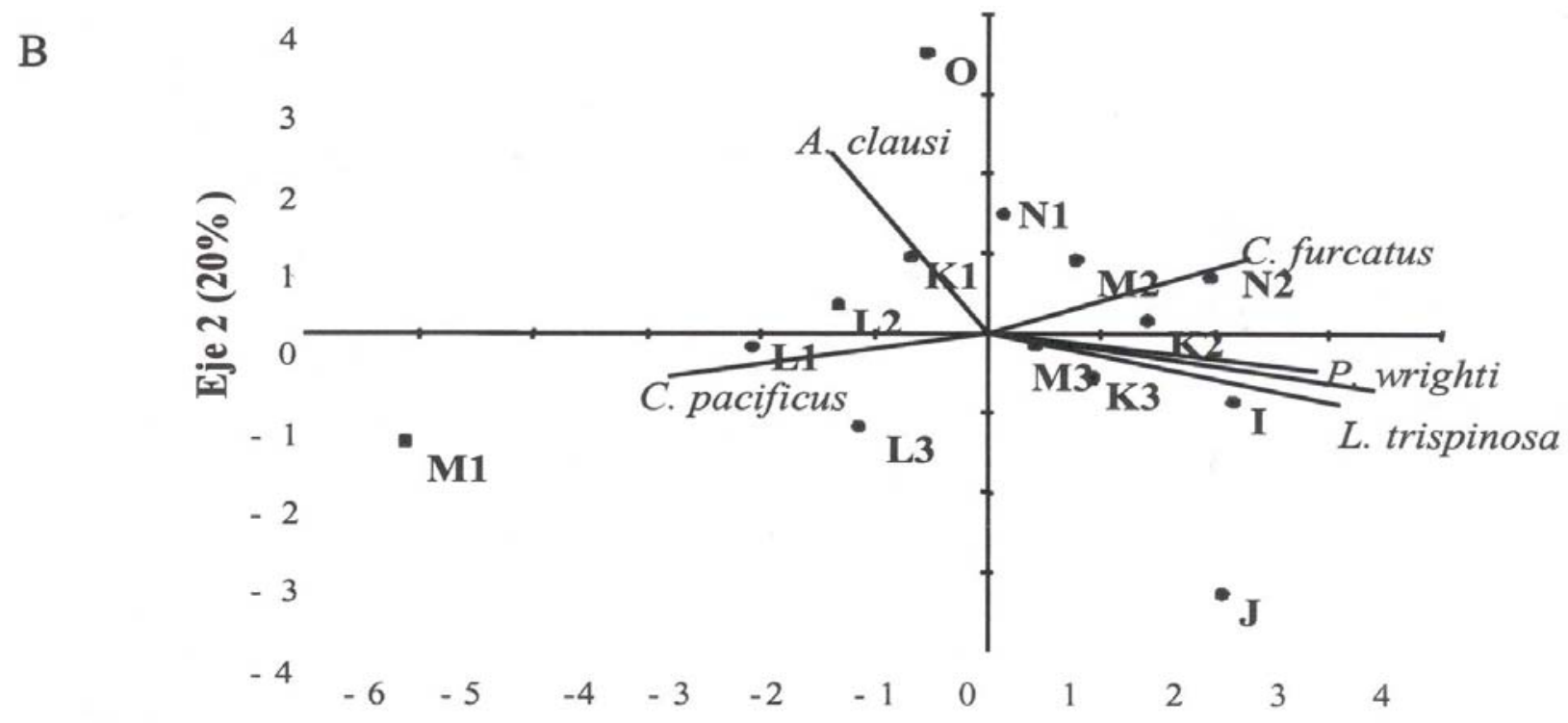

Eje $1(29 \%)$

Figura 7

Biplot de variables (especies) y observaciones (estaciones) de los dos primeros ejes resultantes del análisis de componentes principales en Bahía Magdalena, B. C. S., México. A) mayo 1997, B) agosto 1997, C) noviembre 1997 y D) enero 1998

Biplot of variable (species) and observations (stations) of the two first axis from the Principal Component Analysis in Magdalena Bay, B. C. S., México. A) May 1997, B) August 1997, C) November 1997 and D) January 1998 
C

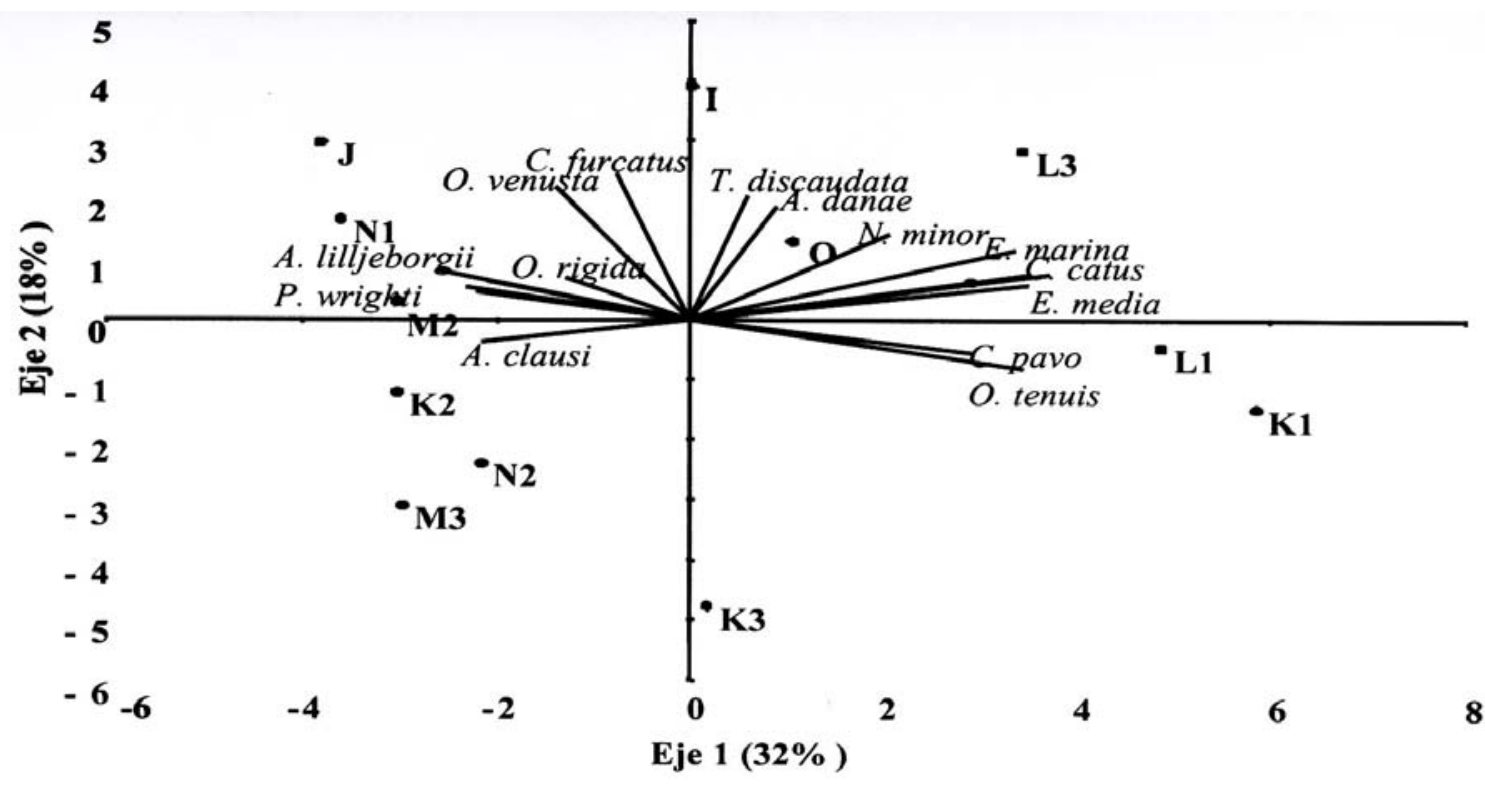

D

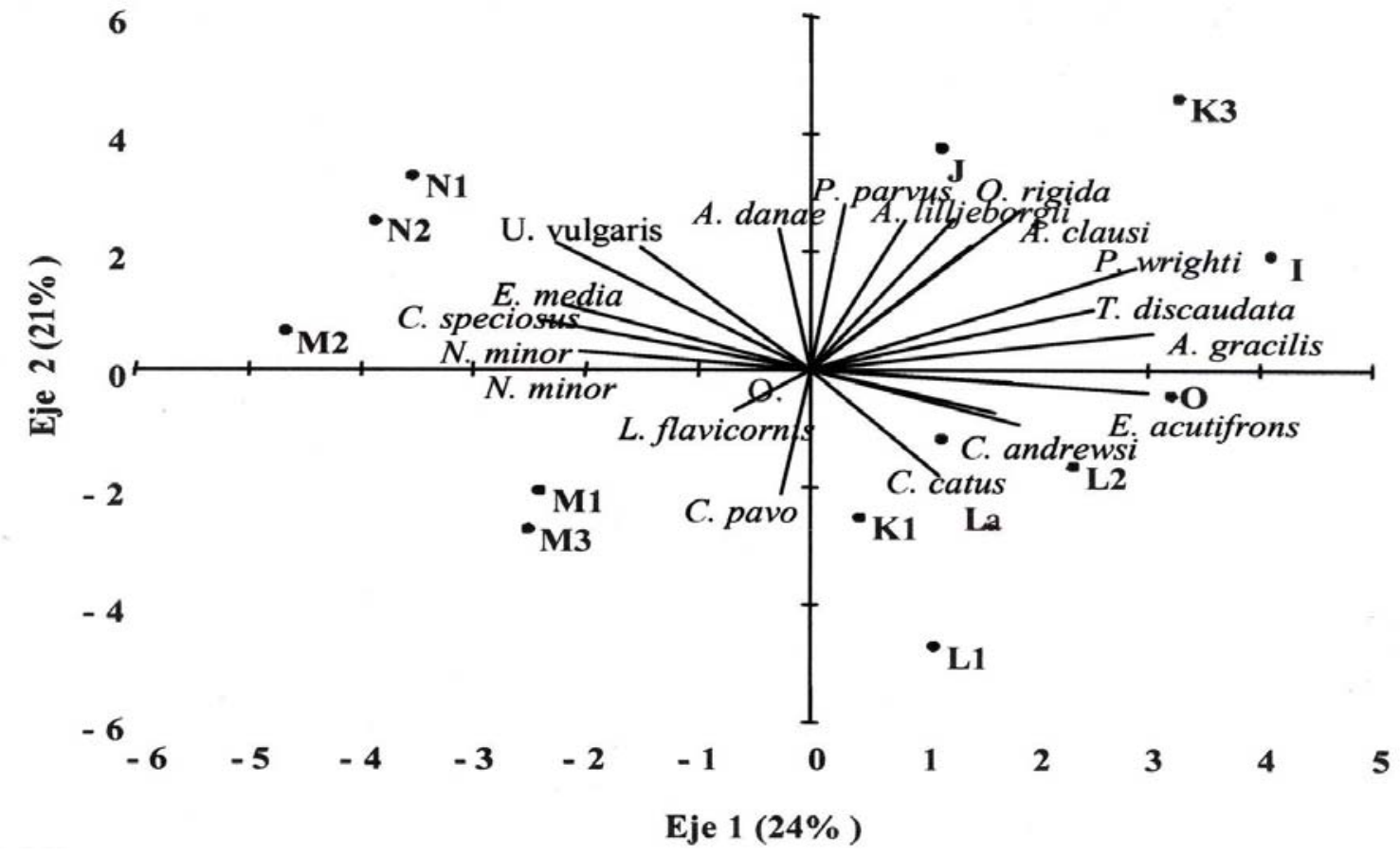

Figura 7 (continuación)

Biplot de variables (especies) y observaciones (estaciones) de los dos primeros ejes resultantes del análisis de componentes principales en Bahía Magdalena, B. C. S., México. A) mayo 1997, B) agosto 1997, C) noviembre 1997 y D) enero 1998

Biplot of variable (species) and observations (stations) of the two first axis from the Principal Component Analysis in Magdalena Bay, B. C. S., México. A) May 1997, B) August 1997, C) November 1997 and D) January 1998 


\section{Discusión}

El evento El Niño 1997/98 ha sido considerado como uno de los eventos más intensos del siglo veinte comparado en magnitud y extensión con El Niño 1983/84 (Lynn et al. 1998, Wolter \& Timlin 1998, McPhaden 1999), a pesar de que tuvo una duración menor (15 meses), fue más intenso que este último, al menos en su etapa inicial. En las costas de Baja California Sur, frente a Bahía Magdalena, El Niño se hizo presente desde junio de 1997 (Gómez-Gutiérrez et al. 1999, Palomares-García et al. 2003), en tanto que en el estado norte (Baja California), la evidencia de El Niño se registró hasta julio del mismo año (Lynn et al. 1998, Lavaniegos-Espejo et al. 2000, LavaniegosEspejo et al. 2002). Nuestros resultados apoyan esta cronología ya que en mayo de 1997, no se registraron anomalías positivas en Bahía Magdalena, en correspondencia con el patrón de temperatura registrado en las aguas adyacentes a la bahía. En cambio, en agosto de 1997, se pudo observar la invasión de aguas cálidas en toda el área, con anomalías de temperatura superiores en $1,2^{\circ} \mathrm{C}$, a las máximas observadas durante el evento de 1982/83. En Bahía Magdalena se registraron anomalías positivas de temperatura, hasta septiembre de 1998, para después entrar en un período de relajación, que culmina con la llegada del evento La Niña en 1999 (Gómez-Gutiérrez et al. 1999, Palomares-García et al. 2003).

Diversos trabajos han relacionado la intensidad de los eventos ENSO con cambios en las comunidades planctónicas. Entre las consecuencias más notables que han sido documentadas se encuentran: la disminución de la productividad primaria y la invasión de especies de afinidad tropical (Fiedler 1984, Barber et al. 1985, Lenarz et al. 1995, Mullin 1995, Lavaniegos-Espejo et al. 2000, Palomares-García \& Gómez-Gutiérrez 1996). Ambos efectos han sido propuestos como los causales directos de la disminución en el volumen de la biomasa zooplanctónica (Chelton et al. 1982, McGowan 1985, Lavaniegos-Espejo et al. 1998, Lavaniegos-Espejo et al. 2001).

Durante mayo, previo a la llegada de El Niño, la biomasa registrada fue la más baja de todo el período de estudio, mientras que en agosto (ya con anomalías positivas en toda la zona), se observaron los valores más altos de biomasa zooplanctónica en la bahía. Uno de los componentes que mayor volumen aportó fue el de los copépodos $\mathrm{y}$, en particular, de los pertenecientes al género Acartia. En noviembre y enero, los valores de biomasa fueron menores que los registrados en agosto. Aunque, en general, los valores observados fueron bajos en comparación con los obtenidos durante principios de la década de los 80s (Palomares-García 1992), el patrón estacional de variación de la biomasa se mantuvo durante los años 1987/88, alcanzando máximo nivel durante el verano (Palomares-García et al. 2003).

La dinámica hidrológica de Bahía Magdalena es determinante en la sucesión de las especies dominantes y también se ve reflejada en la conformación de la estructura de la comunidad. De esta forma, durante mayo de 1997, la presencia de las aguas de la Corriente de California en la zona favoreció la abundancia de especies de afinidad templada (79\%), como Labidocera trispinosa y Calanus pacificus; ésta última considerada como típica de dicha corriente (Hernández-Trujillo 1991, Lavaniegos-Espejo et al. 2000). En agosto y noviembre de 1997, la llegada de aguas inusualmente cálidas favoreció el incremento en el número de especies de afinidad tropical (95\% y $88 \%$ respectivamente), entre las que destaca Pontella fera, especie nerítica de afinidad tropical que no había sido registrada en el interior de la bahía (Hernández-Trujillo et al. 2005), solo había sido reportada para el Golfo de California y Bahía Concepción (Palomares-García et al. 1998). En enero de 1998, las especies más abundantes fueron las del género Paracalanus, siendo P. aculeatus, de afinidad tropical, la mas abundante. Además, se pudo observar que el incremento en el número de especies tropicales se mantuvo (79\%) aunque la temperatura superficial del mar disminuyó.

En la costa oeste de Baja California Sur frente a Bahía Magdalena, Hernández-Trujillo (1999) encontró que la proporción regular de la comunidad de copépodos es tropical en un 50\% en años "no Niño", mientras que en años "Niño" esta fauna se incrementa hasta un 80\%. Durante El Niño 1997/98 se observó un incremento en la abundancia de especies de afinidad tropical o ecuatorial y de ambientes oceánicos mientras que la abundancia de las especies templadas fue menor durante la fase más intensa de El Niño, en la región sur de la Corriente de California (Lavaniegos-Espejo et al. 2000), lo que coincide con lo registrado dentro de Bahía Magdalena. Además, se encontró que las especies templadas como Calanus pacificus registraron abundancias bajas mientras que las especies tropicales y ecuatoriales estuvieron bien representadas, con más de un $80 \%$ de la abundancia total, coincidiendo con lo encontrado por Lavaniegos-Espejo et al. (2000), 
Lavaniegos-Espejo et al. (2002) y Palomares-García et al. (2003). Esta dominancia de las especies de copépodos de afinidad tropical, también coincidió con lo reportado por Palomares-García \& Gómez-Gutiérrez (1996) durante el evento de 1982/83, aunque durante 1982/83 se registró un mayor número de especies (71) mientras que para 1997/98 se registró un total de 66, debido probablemente a que se analizó un número mayor de cruceros y a que este evento duró 8 meses más aproximadamente.

No obstante el incremento en la fauna tropical observada durante el Niño 1997/98, el patrón de sucesión de las especies dominantes en la comunidad de copépodos sufrió pocos cambios con respecto al registrado durante eventos anteriores (Palomares-García 1992, Palomares-García \& Gómez-Gutiérrez 1996). La alternancia de especies del género Acartia por especies del género Paracalanus se mantuvo. Sin embargo, en esta ocasión, Acartia clausi y A. lilljeborgii fueron sustituidas por Paracalanus aculeatus (especie nerítica de afinidad tropical) en vez de $P$. parvus. En estudios anteriores (Palomares-García 1992, Palomares-García \& Gómez-Gutiérrez 1996), la especie de Paracalanus ( $P$. parvus) que reemplazaba a las especies del genero Acartia durante el periodo frío era de afinidad templada, aún bajo condiciones de Niño. La llegada de $P$. aculeatus a esta zona fue uno de los cambios más notables en la composición faunística de Bahía Magdalena, ya que se tiene registro de la permanencia de esta especie en la zona con abundancias elevadas durante los tres años posteriores al evento de El Niño $1997 / 98$, mientras que las poblaciones de $P$. parvus se mantuvieron en bajas densidades (datos sin publicar, CICIMAR). Otra diferencia notable en cuanto a la sucesión de especies es que durante el evento 1997/98 no se presentó la sucesión de A. clausi por A. tonsa; ésta última solo se registró durante enero de 1998, con abundancias muy bajas, mientras que para el evento 1982/83, el reemplazo de $A$. clausi por $A$. tonsa se presentó durante la fase de mayor intensidad de El Niño y posteriormente, $A$. clausi desplazó a $A$. tonsa durante la fase de relajación del evento (Palomares-García \& Gómez-Gutiérrez 1996). Durante El Niño 1997/98, la especie residente $A$. clausi no tuvo una disminución en su abundancia tan acentuada como en 1982/83, por lo que muy probablemente, la población de $A$. tonsa no encontró condiciones favorables al interior de la bahía, posiblemente debido a que su población era muy pequeña como para desarrollarse con éxito y dominar sobre las especies congenéricas, además que $A$. tonsa es más sensible a los cambios de temperatura (Gaudy et al. 2000).

Por otro lado, la influencia de las aguas adyacentes a la bahía se traduce en una separación de ambientes. Hacia el interior de la bahía predominan especies propias de ambientes lagunares y especies que frecuentemente entran a la bahía gracias al intercambio de mareas, en tanto que en el área vestibular son más frecuentes las especies alóctonas propias de ambientes oceánicos y neríticos de afinidad tropical, las cuales presentan sus mayores abundancias en las estaciones cercanas a la boca de la bahía. Es en esta zona donde la influencia de los cambios asociados con eventos El Niño se observa en mayor grado, sobre todo en agosto (fase de mayor intensidad del evento) cuando se presenta una fuerte influencia de aguas tropicales y su fauna asociada. En noviembre y enero, la distribución de estas especies alóctonas abarca una mayor extensión, lo que se refleja en un incremento en la diversidad gracias a la conjunción de especies propias de la bahía y especies que entran a la bahía por el intercambio de mareas. La definición de solo dos grupos de especies de copépodos contrasta con lo observado por PalomaresGarcía (1992) y Palomares-García \& Gómez-Gutiérrez (1996), quienes dividen a Bahía Magdalena en tres zonas, en función del origen de las especies de copépodos observadas en cada una. Estas discrepancias en la definición de ambientes al interior de la bahía puede estar relacionada con el avance de las aguas cálidas y la llegada de especies de origen tropical, lo que favorece una amplia diferencia entre los márgenes internos de la bahía y las aguas del exterior, provocando que no se defina un gradiente semejante al observado en estudios previos.

Las respuestas físicas y biológicas a las condiciones prevalecientes durante El Niño 1997/98, en Bahía Magdalena, fueron distintas a las observadas durante El Niño 1982/83. Durante el primero se alcanzó el valor máximo de temperatura superficial del mar en la zona ( $31^{\circ} \mathrm{C}$ en agosto) y la anomalía de temperatura más elevada $\left(+4,4^{\circ} \mathrm{C}\right)$. Tanto la abundancia específica como la estructura de la comunidad mostraron signos evidentes de perturbación por la llegada de aguas de origen tropical y su fauna asociada; sin embargo, la sucesión de las especies dominantes prevaleció con pocos cambios, a pesar de la magnitud de los cambios ambientales registrados durante El Niño 97/98. 


\section{Agradecimientos}

Los autores agradecen el apoyo financiero de la Dirección de Estudios de Posgrado e Investigación del Instituto Politécnico Nacional (DEPI 20040439) y del Consejo Nacional de Ciencia y Tecnología (CONACyT). A los revisores anónimos por los comentarios y sugerencias para mejorar el manuscrito. G. López Ibarra es becaria PIFI-IPN y del CONACYT y R. Palomares García es becario de los programas COFFA-IPN y EDI-IPN.

\section{Literatura citada}

Álvarez-Borrego S, LA Galindo-Bect \& B Chee-Barragán. 1975. Características hidroquímicas de Bahía Magdalena, B.C.S., México. Ciencias Marinas 2(2): 94-110.

Barber RT, JE Kogelschatz \& PF Chavez. 1985. Origin of productivity anomalies during the 1982-1983 El Niño. California Cooperative Oceanic Fisheries Investigations Reports 26: 65-71.

Brinton R \& JL Reid. 1986. On the effect of interannual variations in circulation and temperature upon euphausiids of California current. En: Pierrot-Bults AC, S Van der Spoel, B Zahuranec \& RK Johnson (eds). Pelagic biogeography, vol. 49: 25-34. UNESCO Technical Paper, Paris.

Chelton DB, PA Bernal \& JA McGowan. 1982. Large-scale interannual physical and biological interaction in the California Current. Journal of Marine Research 40: 10951125.

Fiedler PC. 1984. Some effects of El Niño 1983 on the northern anchovy. California Cooperative Oceanic Fisheries Investigations Reports 25: 53-58.

Fiedler PC, RD Methot \& RP Hewitt. 1986. Effects of California El Niño 1982-1984 on the northern anchovy. Journal of Marine Research 44: 317-338.

Gaudy R, G Cervetto \& M Pagano. 2000. Comparison of the metabolism of $A$. clausi and $A$. tonsa: Influence of temperature and fsalinity. Journal of Experimental Marine Biology and Ecology 247: 51-65.

Gómez-Gutiérrez J, R Palomares-García \& D Gendron. 1995. Community structure of the euphausiid populations along the west coast of Baja California, Mexico during the weak ENSO 1986-1987. Marine Ecology Progress Series 120: 41-51.

Gómez-Gutiérrez J, R Palomares-García, R De SilvaDavila, A Carballido-Carranza \& A Martinez-López. 1999. Copepod daily egg production and growth rates in Bahía Magdalena, Mexico. Journal of Plankton Research 21(12): 2227-2244.
Hernández-Trujillo S. 1991. Patrones de distribución y abundancia de Calanus pacificus en relación a la temperatura superficial en el Pacífico de Baja California Sur, México (1982-1986). Revista Investigación Científica 2(1): 56-64.

Hernández-Trujillo S. 1999. Variability of community structure of Copepoda related to El Niño 1982-83 and 1987-88 along the west coast of Baja California Peninsula, Mexico. Fisheries Oceanography 8(4): 1-12.

Hernández-Trujillo S, R Palomares-García, GA LópezIbarra, G Esqueda-Escárcega \& R Pacheco-Chávez. 2005. Riqueza específica de copépodos en Bahía Magdalena, Baja California Sur, México. Anales del Instituto de Biología, Universidad Nacional Autónoma de México, Serie Zoología 75(2): 253-270.

Lavaniegos-Espejo B, J Gómez-Gutiérrez, JR Lara-Lara \& S Hernández-Vázquez. 1998. Long-term changes in zooplancton volumes in the California Current System the Baja California region. Marine Ecology Progress Series 169: 55-64.

Lavaniegos-Espejo B, B Gaxiola, G Jiménez, L González, M Baumgartner, T Durazo, R García \& J Lara. 2000. Efectos del evento climático El Niño 1997/98 sobre el ecosistema pelágico de la Corriente de California. Simposio CONACyT. Los efectos del fenómeno El Niño en México. México, D. F. 17-19 de mayo de 2000. Pp. 147-163.

Lavaniegos-Espejo B, LC Jiménez-Pérez \& G GaxiolaCastro. 2002. Plankton response to El Niño 1997-1998 and La Niña 1999 in the southern region of the California Current. Progress in Oceanography 54: 33-58.

Lenarz H, A Ventresca, M Graham, B Schwing \& $F$ Chavez. 1995. Explorations of El Niño events and associated biological population dynamics off Central California. California Cooperative Oceanic Fisheries Investigations Reports 36: 106-119.

Lynn RJ, T Baumgartner, J García, J, CA Collins, TL Hayward, KD Hyrenbach, AW Mantyla, T Murphree, A Shankle, FB Schwing, KM Sakuma \& MJ Tegner. 1998. The state of the California Current, 1997-1998: transition to El Niño conditions. California Cooperative Oceanic Fisheries Investigations Reports 39: 25-49.

McGowan JA. 1985. El Niño 1983 in the Southern California Bight. En: Wooster WS \& DL Fluharty (eds). El Niño North. El Niño effects in the eastern subarctic Pacific Ocean, pp. 66-184. Washington Sea Grant Program, University of Washington.

McPhaden MJ. 1999. Genesis and evolution of the 1997-98 El Niño. Science 283: 950-954. 
Miller CB, RD Batchelder, RD Brodeur \& WG Pearcy. 1985. Response of the zooplankton and ichthyoplankton off Oregon to the El Niño of 1983. En: Wooster WS \& DL Fluharty (eds). El Niño North. El Niño effects in the eastern subarctic Pacific Ocean, pp. 185-187. Washington Sea Grant Program, University of Washington.

Mullin M. 1995. The Californian El Niño of 1992 and the fall of Calanus. California Cooperative Oceanic Fisheries Investigations Reports 36: 175-178.

Palomares-García R. 1992. Analysis of the community of copepods in the lagoon complex of Magdalena-Almejas Bay, B.C.S., in 1985 - 1986. Ciencias Marinas 18: 71-92.

Palomares-García R \& J Gómez-Gutiérrez. 1996. Copepod community structure at Bahia Magdalena, México during El Niño 1983 - 1984. Estuarine, Coastal and Shelf Science 43: 583-595.

Palomares-García R, E Suárez \& S Hernández. 1998. Catálogo de los copépodos (Crustacea) pelágicos del Pacífico mexicano. Coedición CICIMAR-IPN, ECOSUR, 352 pp.

Palomares-García R, R De Silva, A Martínez, A Hinojosa, R Avendaño \& R Funes. 2001. El evento El Niño 19971998 y su impacto sobre el zooplancton en Bahía Magdalena, B.C.S. En: Consejo Nacional de Ciencia y Tecnología (ed). Los efectos del fenómeno El Niño en México 1997-1998. 1: 192-198.
Palomares-García R, R De Silva, A Martínez, R Funes, A Carballido, R Avendaño, A Hinojosa \& GA LópezIbarra. 2003. Biological effects of El Niño 1997/98, in a shallow subtropical ecosystem. Geofísica Internacional 42(3): 455-466.

Pielou EC. 1975. Ecological diversity. 627 pp. Wiley Interscience, New York.

Raymont JEG. 1983. Plankton and Productivity in the Oceans. $2^{\text {nd. }}$ ed, vol. 2. Zooplankton, 824 pp. Pergamon Press, Gran Bretaña.

Roden G. 1971. Aspect of the transition zone in the Northeastern Pacific. Journal of Geophysical Research 5: 3462-3475.

Stedman HF. 1976. Zooplankton fixation and preservation. Monographs on Oceanographic Methodology 4, 174 pp. UNESCO Press, Paris.

Wolter K \& MS Timlin. 1998. Measuring the strength of the ENSO events: How does 1997/98 rank? Weather 53: 315324. 